\title{
Effects of Dietary Fiber from Corn Husks on Hyperlipidemia prevention in Trans-fatty Acids fed Mice
}

\author{
Fan $\mathrm{Xu}^{1}$, Yazhe Xiao ${ }^{1}$, Mingfei $\mathrm{Xu}^{1}$, and Jia Liu ${ }^{1, *}$ \\ ${ }^{1}$ School of Basic Medicine of Changsha Medical University, Changsha, Hunan, 410219, China
}

\begin{abstract}
To evaluate the effects of dietary fiber from corn husks on hyperlipidemia, the hyperlipidemia model of mice was induced by trans-fatty acids diet,and the mice were randomly divided into the blank control group, the high fat control group(TFA group) and the low, middle and high dose group (2.5,5.0,10.0 $\mathrm{G} / \mathrm{kg}$ ) of dietary fiber from corn husks. Except the blank control group, the mice were fed with high fat diet. The body mass, serum total cholesterol, triglyceride, low density lipoprotein(LDL) and high density lipoprotein(HDL) were measured. The intake of trans-fatty acids resulted in a significant increase in the body weight, serum total cholesterol, triglyceride and $\operatorname{LDL}(P<0.01)$, HDL level was significantly lower $(P<0.01)$. Corn husks dietary fiber intervention can reduce the body weight of mice, the serum total cholesterol, triglyceride, LDL concentration and increase HDL concentration. The difference between the high-dose group and the TFA group was significant $(P<0.01)$, and the intervention effect was best in the group with high dose of corn husks dietary fiber. Corn husks dietary fiber can effectively reduce blood lipid in hyperlipidemia mode mice.
\end{abstract}

\section{1 introduction}

Hyperlipidemia, also known as dyslipidemia or lipid metabolic disorder, refers to high serum cholesterol, triglyceride, Low-density Lipoprotein(LDL) and low high-density Lipoprotein(HDL). It is one of the important risk factors of cardiovascular and cerebrovascular diseases ${ }^{[1-2]}$. The cause of hyperlipidemia is due to deficiency of fat metabolism, improper diet and excess of food. In traditional Chinese medical theory, it is the causes including stagnation of phlegm or liver, and blood stasis. All kinds of causes lead to the pathological products cross-block ${ }^{[3]}$. In the world, about 17 million people die from cardiovascular disease each year and 23.6 million are expected to die by 2030 , according to the report ${ }^{[4]}$. At present, there are many kinds of chemical drugs for hyperlipidemia at home and abroad, mainly including statins, betates and niacin. Statins are the first choice. They have strong lipid-lowering effect and clear mechanism of action, but they may cause adverse reactions such as liver injury, muscle disease, skin allergy, digestive system and urinary system diseases ${ }^{[5-6]}$. Studies have shown that insufficient dietary fiber intake is an important factor in the development of such disease ${ }^{[7]}$. The yield of corn in China is high, and the corn husks is rich in dietary fiber. The research showed that dietary fiber in corn husks has the pharmacological effects of reducing blood pressure, lowering energy value, promoting intestinal patency and preventing cardiovascular disease. In this experiment, the hyperlipidemia model mice were established, and the corn husks was used as the raw material to prepare the dietary fiber, and the hyperlipidemia model mice were given low, middle and high doses dietary fiber diet, serum TC, TG, HDL-C and LDL-C were measured by enzyme-linked immunosorbent Assay (Elisa) to study the effect of dietary fiber from corn husks on the level of blood lipid in hyperlipidemia mice induced by trans-fatty acids.

\section{Materials and methods}

\subsection{Experiment materia}

Experimental animals: 40 healthy and clean SPF KM mice, male or female, weighing $(20 \pm 2)$ g were provided by Changsha Tianqin Biotechnology company. All mice were raised in the standardized laboratory of Changsha Medical University. Mice were allowed to eat and drink freely before the experiment and acclimatize to the environment for at least a week before the experiment.

Experimental supplies: HDL Kit, LDL Kit, Total cholesterol Kit, triglyceride kit were provided by Nanjing JianCheng Institute of Biological Engineering. Water soluble corn husks dietary fiber (SDF) made in laboratory.

\footnotetext{
* Corresponding author: forwork99@sina.com
} 


\subsection{Experimental procedure}

\subsubsection{Modeling method of hyperlipidemia mice}

High-fat model ${ }^{[8]}$, known as $10 \%$ Trans Fatty Acid (TFA) model, was used in the modeling of hyperlipidemia mice [9]. High cholesterol and bile salts were added to the model feed. In addition to the blank control group, the other groups were fed with a high-fat diet consisting of $10 \%$ yolk powder, $0.5 \%$ bile salt, $5 \%$ lard and basic feed to induce hyperlipidemia.

\subsubsection{Model detection index}

After one week of adaptive feeding, the body mass of mice was measured and recorded daily. Four weeks later, the mice were fasting for $24 \mathrm{~h}$, and the blood samples were taken from the orbit to measure the serum total cholesterol(TC),triglyceride(TG),high-density

lipoprotein cholesterol (HDL-C) and low-density lipoprotein cholesterol (LDL-C).The mice were free to drink and feed during the experiment. When the serum total cholesterol TC $>5.72 \mathrm{Or}$ serum triglyceridesTG $>1.70$ $(\mathrm{mmol} / \mathrm{L})$ or $\mathrm{TC}>5.72(\mathrm{mmol} / \mathrm{L})$ and $\mathrm{TG}>1.70(\mathrm{mmol} / \mathrm{L})$ Or HDL cholesterolHDL-C $<0.9(\mathrm{mmol} / \mathrm{L})^{[6]}$, the models were made successfully.

\subsubsection{Experiment in groups}

blank control group: Regular feed;

High fat control group (TFA group): High-fat feed was given;

Corn husks dietary fiber low dose group: The low-dose corn husks group was given 10\%TFA feed and corn husks dietary fiber, dose $2.5(\mathrm{~g} / \mathrm{kg})$.

Corn husks dietary fiber middle dose group: The medium dose corn husks group was given 10\%TFA feed and corn husks dietary fiber, dose $5.0(\mathrm{~g} / \mathrm{kg})$.

Corn husks dietary fiber high dose group: The high-dose corn husks group was given 10\%TFA feed and corn husks dietary fiber, dose $10.0(\mathrm{~g} / \mathrm{kg})$.

All feed supplies were for four consecutive weeks.

\subsubsection{Measurement and calculation method of each index}

Body mass measurement: The body mass of mice was weighed and recorded every Sunday morning before feeding.

Determination of serological indexes: after fasting for $24 \mathrm{~h}$, blood was taken from the orbit of mice, centrifuged, and supernatant was taken, according to the kit method. Serum total cholesterol TC triglyceride TG high density lipoprotein cholesterol HDL-C and low density lipoprotein cholesterol LDL-C were measured.

\subsection{5 data statistics}

All values were expressed as means \pm SD. Differences among several groups were determined by one-way ANOVA; comparisons between groups were carried out by the LSD t-test, using SPSS software (version 17.0). $P<0.05$ was considered to be statistically significant.

\section{Results}

\subsection{Basic information of mice}

In the blank control group, the mice had good mental condition, sensitive reaction, free movement, shiny fur, and good fluidity of plasma collected from blood collection. During the period of high-fat feed feeding, mice in the high-fat group were fat, listless, lethargic, and dull fur, and the blood plasma collected was sticky and poor fluidity. During the feeding of corn husks dietary fiber, mice in the middle and high dose groups of corn husks dietary fiber gradually returned to normal behavior.

\subsection{Volume mass change}

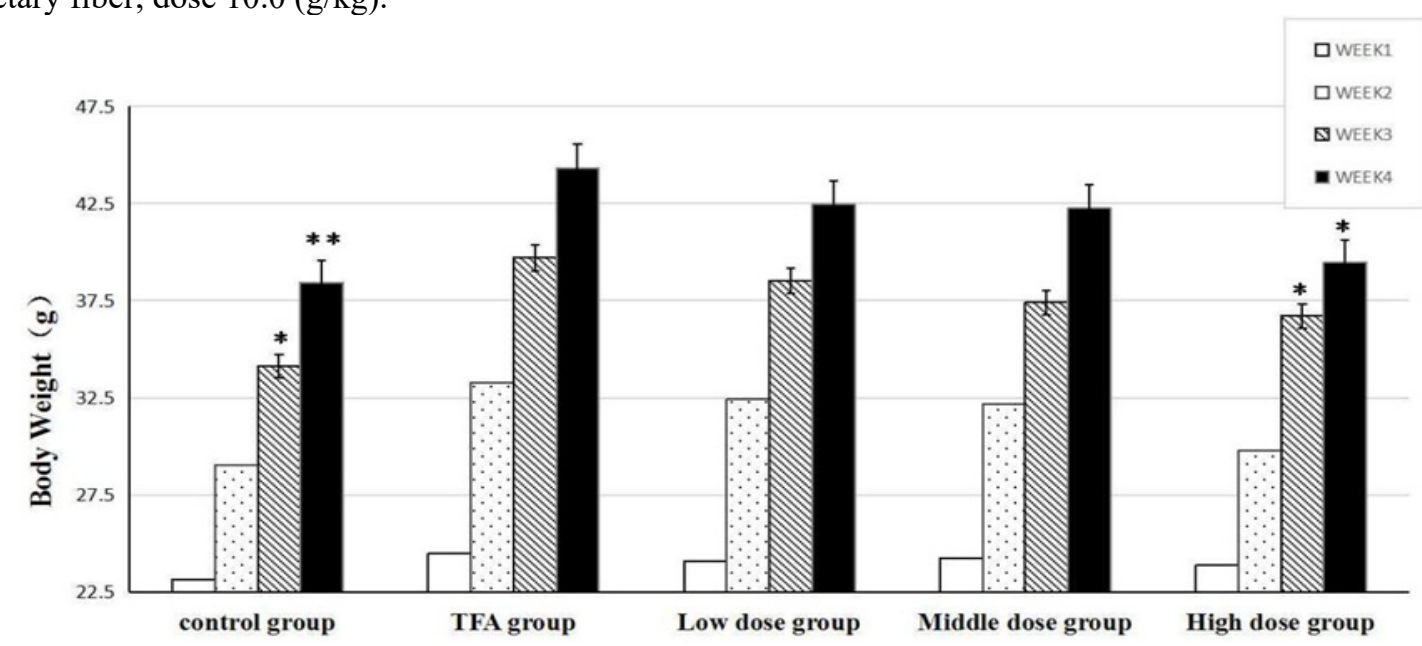

Figure.1. diagram one Effects of dietary fiber from corn husk on body quality in mice

As can be seen from Figure 1, after modeling, the mice in the high-fat group (TFA group) gained significantly more weight than the blank control group $(P<0.01)$. It can be seen that a long-term high-fat diet can affect 
weight change. After four weeks of continuous feeding of corn husks dietary fiber, the body weight of the low dose and middle dose group decreased, while that of the high-dose group decreased significantly $(P<0.01)$. It suggested that dietary fiber from corn husks could inhibit the weight gain of hyperlipidemia mice.

\subsection{Changes of TC, TG and LDL-C in serum of mice}

It can be seen from Table 1, the serum TC, TG and LDL$\mathrm{C}$ of the TFA group were significantly higher than those of the blank control group $(\mathrm{P}<0.01)$. The results showed that the high-fat diet could significantly increase the levels of TC, TG and LDL-C in serum of mice, and the model was successfully constructed. Compared with the TFA group, the dietary fiber of corn husks decreased to different degrees in each dose intervention group, and the difference between the high-dose group and the TFA group was significant $(P<0.01)$. The results showed that high dose dietary fiber intake of corn husks was beneficial to the reduction of blood lipid, and the effect was better than that of middle dose and low dose groups.

Table.1. Effect of dietary fiber from corn husk on lipid levels $(\mathrm{n}=8 \quad \mathrm{x} \pm \mathrm{s})$

\begin{tabular}{|c|c|c|c|}
\hline group & $\mathrm{TC} /(\mathrm{mmol} / \mathrm{L})$ & $\begin{array}{c}\mathrm{TG} /(\mathrm{mmol} / \mathrm{L} \\
)\end{array}$ & $\begin{array}{c}\text { LDL- } \\
\mathrm{C} /(\mathrm{mmol} / \mathrm{L})\end{array}$ \\
\hline Control group & $2.39 \pm 0.16^{\triangle \triangle}$ & $1.75 \pm 0.13^{\triangle \triangle}$ & $0.74 \pm 0.33^{\triangle \triangle}$ \\
\hline TFA group & $7.48 \pm 0.50$ & $3.05 \pm 0.34$ & $2.16 \pm 0.26$ \\
\hline Low dose group & $6.20 \pm 0.73$ & $2.37 \pm 0.23^{\triangle}$ & $1.88 \pm 0.24$ \\
\hline $\begin{array}{c}\text { Middle dose } \\
\text { group }\end{array}$ & $6.03 \pm 0.36^{\triangle}$ & $1.98 \pm 0.19^{\triangle}$ & $1.45 \pm 0.30^{\triangle}$ \\
\hline High dose group & $4.88 \pm 0.33^{\triangle \triangle}$ & $1.85 \pm 0.15^{\triangle \triangle}$ & $1.05 \pm 0.24^{\triangle \triangle}$ \\
\hline
\end{tabular}

Note: Comparison with high-fat group, ${ }^{\triangle}$ : $(P<0.05)$; ${ }^{\triangle \Delta}$; $(P<0.01)$

\subsection{Changes of serum HDL-C in mice}

It can be seen from Table 2, the serum HDL-C content in the TFA group was significantly lower than that in the blank control group $(\mathrm{P}<0.01)$. The results showed that the high fat feed, especially the high fat feed containing TFA, could significantly reduce the serum HDL-C content of mice. As can be seen from Table 2, compared with the high-fat group, all dietary fiber intervention groups could increase the serum HDL-C content of mice to different degrees, among which the high-dose dietary fiber could significantly increase the HDL-C level of mice $(\mathrm{P}<0.01)$, which make their HDL-C levels similar to those of the blank control group. Overall, the higher the amount of dietary fiber added, the higher the serum HDL-C content.
Table.2. Effect of dietary fiber from corn husk on HDL levels $(\mathrm{n}=8 \quad \mathrm{x} \pm \mathrm{s})$

\begin{tabular}{|c|c|c|}
\hline group & $\begin{array}{c}\text { Dose }(\mathrm{g} / \mathrm{kg} . \\
\text { bw) }\end{array}$ & HDL-C/(mmol/L) \\
\hline Control group & $/$ & $2.21 \pm 0.16^{\triangle}$ \\
\hline TFA group & $/$ & $0.88 \pm 0.38$ \\
\hline Low dose group & 2.5 & $1.21 \pm 0.26$ \\
\hline Middle dose group & 5.0 & $1.59 \pm 0.26$ \\
\hline High dose group & 10.0 & $2.30 \pm 0.46^{\triangle}$ \\
\hline
\end{tabular}

Note: Comparison with high-fat group, $\triangle: P<0.01$

\section{Discuss}

The reason for the increase of $\mathrm{TC}$ and $\mathrm{TG}$ in hyperlipidemia may be that certain groups in TFA are more likely to bind to enzyme activities in the small intestine and are hydrolyzed into small molecules, which then bind to phospholipid cholesterol in the small intestine, resulting in the increase of cholesterol and triglyceride content in the blood. Under normal circumstances, most bile acids are reabsorbed in the small intestine through the hepatoenteric circulation into the liver to keep the bile acid pool stable. In fat metabolism, corn husks dietary fiber interferes its reabsorption by absorbing bile acid and reduces the solubility of molecular groups of cholesterol and triglyceride digestion products, thus inhibiting or delaying the absorption of cholesterol and triglyceride.

The mechanism of trans fatty acids decreasing HDL$\mathrm{C}$ and increasing LDL-C may be related to cholesterol ester transporter protein (CETP), whose function is to transport cholesterol esters from HDL-C to very low density lipopro-tein (VLDL) and LDL. It plays an important role in the reverse transport of cholesterol and the function of corn husks dietary fiber to increase HDL$\mathrm{C}$ and decrease LDL-C may be related to apolipoprotein plasma. In this study, it can be seen that dietary fiber from corn husks can effectively regulate blood lipid level in hyperlipidemia mice by reducing TC content and inhibiting LDL-C level, which has certain effects on lipid reduction. The study and specific mechanism of dietary fiber from corn husks in diseases related to lipid metabolism need to be further studied.

\section{Acknowledgement}

This work was supported by the Hunan Natural Science Foundation Youth Project (201910823015).

\section{References}

1. Nelson R H. Hyperlipidemia as a risk factor for cardiovascular disease. Prim Care, 40(1):195. (2013)

2. Reimann $M$, Rudiger $H$, Weiss $N$, et al. Acute hyperlipidemia but not hyperhomocysteinemia impairs reflex regulation of the car-diovascular system. Atheroscler Suppl, 18:8. (2015) 
3. JW Liang. Etiology and Pathogenesis of hyperlipidemia in Chinese medicin. Chinese Journal for Clinicians, 40(3):23. (2012)

4. World Health Organization. The top 10 causes of death [EB/OL]. www.who.int/mediacentre.

5. Bjornsson E, Jacobsen E I, Kalaitzakis E. Hepatotoxicity associ-ated with statins: reports of idiosyncratic liver injury post-market-ing. J Hepatol, 56(2): 374.(2012)

6. Norata G D, Tibolla G, Catapano A L. Statins and skeletal mus-cles toxicity: from clinical trials to everyday practice. Pharma-col R es, 88:107. (2014)
7. JJ Liu, LQ Jia, MJ Lv, GL Yang. Research progress of hyperlipidemia combined Chinese and Western medicine, 35(04):815-818. (2017)

8. SQ Zhang, L Tong. Research progress of insulin resistance mechanism and laboratory model. Tradit Chin Drug Res Pharmacol, 23(3)365-366(2012)

9. The effect of dietary fiber of corn husk on lipid and antioxidant capacity in mice with hyperlipidemia induced by trans fatty acids [D]. Wuhan: Master's thesis of Wuhan Institute of Technology, (2011). 